\title{
Application of Fractional Order PID Controller to a DC Motor System
}

\author{
Tung-Yung Huang
}

\begin{abstract}
PID controllers have been successfully used in versatile applications, especially with the aid of available mature tuning methods such as the Ziegler-Nichols method. Recently, fractional order PID controllers were proposed after a series of researches on fractional calculus. It is intriguing for the flexibility of the adoption of fractional order which is free from the limit of integer order. This paper studied the fractional order PID controller of different fraction order applied in a DC motor system. The simulation results show that the fractional order PID controllers are promising compared to the integer PID controllers.
\end{abstract}

Keywords—fractional order, PID, parameter tuning

\section{Introduction}

The application using fractional calculus initiated in early 19th century [1]. Abel used fractional calculus to formulate and solve the "tautochrone" problem. Later on, Liouville, Heaviside and other mathematicians derived mathematical models for some systems and solved those problems with the fractional calculus. These systems included potential theory, heat equation, and notch design of a dam. Then the research was dormant for quite a while until the late 20th century. There were consecutively three International Conferences on Fractional Calculus held in University of New Haven in Connecticut, USA, Strathclyde University in Scotland, England and Nihon University in Tokyo, Japan. Researchers presented miscellaneous fractional order systems, including rheology, diffusion, electromagnetic theory, statistics, probability, viscoelasticity and electrochemistry of corrosion.

Commonly-used PID controller uses the linear combination of the input value, and its derivative and integral as the output. Its simplicity, effectiveness and robustness makes it popular in industrial applications. In addition, the parameters tuning methods, such as the Ziegler-Nichols method, which require only time response without parametric system models make the PID controllers even more convenient and effective.

Compared to conventional (integer) PID controllers, though fractional order PID controllers seem to be suitable for

Tung-Yung Huang / Assistant Professor

Department of Mechanical Engineering

Shih-Ying Hung / Graduate student

Southern Taiwan University of Science and Technology

Tainan City, 71005 Taiwan.
Shih-Ying Hung

intrinsically fractional order dynamic systems, they do not receive much attention until recent years due to its complexity and lack of parameters tuning algorithms.

Monje et al [2] proposed to optimize parameters of fractional order PID controllers by using gain crossover frequency, phase margin and other conditions in 2004. Yet, this method is not well developed. The initial guess must be fairly good so the parameters may converge to optimum. Valério and Sá da Costa [3] proposed a parameters tuning algorithm for fractional order PID controllers adhering to the concept of the Ziegler-Nichols method in 2005. However, it's useful only for S-shape step response with time delay, not suitable for system response without time delay.

This paper studied the feasibility of applying the $\mathrm{PI}^{\lambda} \mathrm{D}^{\mu}$ (fractional PID, $0 \leq \lambda, \mu$ ) controller to a DC motor system. The Ziegler-Nichols method provides a clue for determining the parameters of the $\mathrm{PI}^{\lambda} \mathrm{D}^{\mu}$ controller as there is no mature tuning method yet. Several parameter sets were tried for comparsion. It is desired to find useful $\mathrm{PI}^{\lambda} \mathrm{D}^{\mu}$ controller for the specified system.

This paper consists of the following sections: section I is the introduction, section II presents the mathematic model of a DC motor system, section III briefly introduces the fractional calculus, and fractional order Laplace transform, section IV gives the simulation results, and section $\mathrm{V}$ is the conclusion.

\section{DC Motor Model}

A DC motor model is used as an example (Fig. 1). The armature voltage is $e(t)$, and the current through armature is $i(t)$. The governing electrical equation is

$$
e(t)=L_{a} \frac{d i(t)}{d t}+R_{a} i(t)+e_{b}(t)
$$

where $L_{a}$ and $R_{a}$ are the inductance and resistance of the armature, respectively. And $e_{b}(t)$ is the back e.m.f. of the DC motor, which is proportional to the angular velocity $\omega$. Let the back e.m.f. constant be $K_{b}$, then

$$
e_{b}(t)=K_{b} \omega(t)=K_{b} \frac{d \theta(t)}{d t}
$$

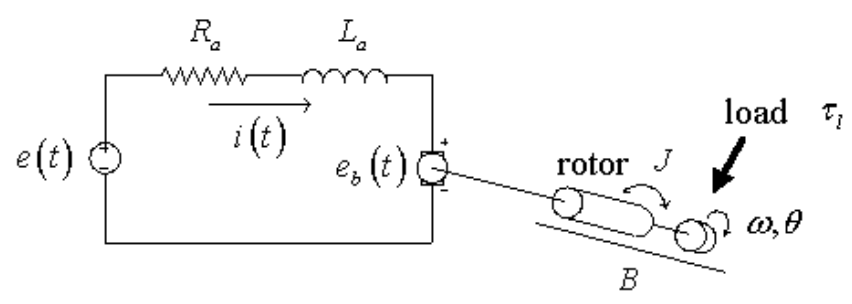

Figure 1. DC Motor 
Meanwhile, assume the generated motor torque and the load are $\tau_{m}$ and $\tau_{l}$, respectively. The rotational inertia and the rotational damping coefficient of the motor are $J$ and $B$, respectively. Then the governing mechanical equation can be expressed as

$$
\tau_{m}(t)=J \frac{d^{2} \theta(t)}{d t^{2}}+B \frac{d \theta(t)}{d t}+\tau_{i}(t)
$$

Since the motor's output torque is proportional to the input current, it is usually described by the following equation

$$
\tau_{m}(t)=K_{t} i(t)
$$

where $K_{t}$ is the torque constant. When the SI system is used, $K_{b}$ equals $K_{t}$.

The DC motor system can be represented by the block diagram shown in Fig. 2. Hence, the transfer function of the DC motor using the Laplace transform is

$$
G(s)=\frac{\Theta(s)}{E(s)}=\frac{K_{t}}{s\left[(J s+B)\left(L_{a} s+R_{a}\right)+K_{t} K_{b}\right]}
$$

if the rotation angle is chosen as output.

$$
G_{\Omega}(s)=\frac{\Omega(s)}{E(s)}=\frac{K_{t}}{\left[(J s+B)\left(L_{a} s+R_{a}\right)+K_{t} K_{b}\right]}
$$

if the angular velocity is chosen as output.

Using the motor parameters in Table I, the transfer function is

$$
\frac{\Omega(s)}{E(s)}=\frac{0.01826}{3.378 \times 10^{-7} s^{2}+1.848 \times 10^{-4} s+5.673 \times 10^{-4}}
$$

\section{Fractional Order Controller}

\subsection{Fractional Calculus}

Fractional calculus operator is defined as follows [4]:

TABLE I.

DC MOTOR PARAMETERS

\begin{tabular}{|l|l|l|}
\hline \multicolumn{1}{|c|}{ Physical quantity } & \multicolumn{1}{|c|}{ Symbol (unit) } & \multicolumn{1}{c|}{ value } \\
\hline Armature inductance & $L_{a}(\mathbf{H})$ & $4.6909 \times 10-3$ \\
\hline Armature resistance & $R_{a}(\mathbf{\Omega})$ & 2.5604 \\
\hline Back e.m.f. constant & $K_{b}(\mathrm{~V} /(\mathrm{rad} / \mathrm{s}))$ & $1.8259 \times 10-2$ \\
\hline Torque constant & $K_{t}(\mathbf{N} \cdot \mathbf{m} / \mathbf{A})$ & $1.8259 \times 10-2$ \\
\hline Damping coefficient & $B(\mathbf{N} \cdot \mathbf{m} /(\mathrm{rad} / \mathrm{s}))$ & $9.1358 \times 10-5$ \\
\hline Rotational inertia & $J\left(\mathrm{~kg} \cdot \mathbf{m}^{2}\right)$ & $7.2019 \times 10-5$ \\
\hline
\end{tabular}

Figure 2. Block diagram of a DC motor system

$$
{ }_{a} D_{t}^{\alpha}=\left\{\begin{array}{cc}
\frac{d^{\alpha}}{d t^{\alpha}} & \operatorname{Re}(\alpha)>0 \\
1 & \operatorname{Re}(\alpha)=0 \\
\int_{a}^{t}(d \tau)^{-\alpha} & \operatorname{Re}(\alpha)<0
\end{array}\right.
$$

where $t$ and $a$ are the upper limit and lower limit of the integral, respectively. And $\alpha$ is the fractional order. If $\alpha>0$, ${ }_{a} D_{t}^{-\alpha}$ is a fractional order integral, and ${ }_{a} D_{t}^{\alpha}$ is a fractional order differential. The most famous fractional order integral definition is the Riemann-Liouville definition, which is expressed as follows [4]:

$$
{ }_{a} D_{t}^{\alpha} f(t)=\frac{1}{\Gamma(\alpha)} \int_{a}^{t}(t-\tau)^{\alpha-1} f(\tau) d(\tau)
$$

where

$$
\Gamma(x)=\int_{0}^{\infty} y^{x-1} e^{-y} d y
$$

is the well-known Euler's gamma function. While the most used fractional order differential definition is the GrünwaldLetnikov definition, which is expressed as follows [4]:

$$
{ }_{a} D_{t}^{\alpha} f(t)=\lim _{\substack{h \rightarrow 0 \\
n h=t-a}} h^{-\alpha} \sum_{r=0}^{n}(-1)^{r}\left(\begin{array}{c}
\alpha \\
j
\end{array}\right) f(t-r h)
$$

where

$$
\left(\begin{array}{l}
\alpha \\
0
\end{array}\right)=1,\left(\begin{array}{l}
\alpha \\
r
\end{array}\right)=\frac{\alpha(\alpha-1) \cdots(\alpha-r+1)}{r !}
$$

Since Laplace transform is used to solve engineering problems described by ordinary differential equations, it can be used in fractional order differointegral equations as well.

The Laplace transform according to the Riemann-Liouville definition is

$$
L\left\{{ }_{0} D_{t}^{\alpha} f(t)\right\}=s^{\alpha} F(s)-\sum_{j=0}^{n-1} s^{j}\left[{ }_{0} D_{t}^{\alpha-j-1} f(0)\right]
$$

when $(n-1) \leq \alpha<n$

$$
\begin{aligned}
& \text { If }{ }_{0} D_{t}^{\alpha-j-1} f(0)=0, j=0,1,2, \cdots n-1 \\
& \text { then } L\left\{{ }_{0} D_{t}^{\alpha} f(t)\right\}=s^{\alpha} F(s)
\end{aligned}
$$

Obviously, the order of the Laplace operator $s$ is a fraction in fractional order differointegral.

Oustalop [5] proposed to apply fractional order controllers to dynamic systems. He named the non-integer robust controller he developed as "Commande Robuste d'Ordre Non Entier" (CRONE). The implementation was described in his book, and various application examples were provided therewith. He later introduced another fractional order PID controller, or the $\mathrm{PI}^{\lambda} \mathrm{D}^{\mu}$ controller. The transfer function of such a controller takes the following form 


$$
G_{C}(s)=K_{P}+\frac{K_{I, \lambda}}{s^{\lambda}}+K_{D, \mu} s^{\mu} \quad(\lambda, \mu>0)
$$

where $K_{P}, K_{I, \lambda}$, and $K_{D, \mu}$ are the proportional gain, the fractional order integral gain, and the fractional order derivative gain, respectively.

And the control effort $u(t)$ in time domain can be expressed as

$$
u(t)=K_{P} e(t)+K_{I, \lambda} D^{-\lambda} e(t)+K_{D, \mu} D^{\mu} e(t)
$$

When $\lambda=\mu=1$ in equation (3-7), $G_{C}(s)$ is an integer PID controller; $\lambda=1, \mu=0, G_{C}(s)$ a PD controller; and $\lambda=0, \mu=1, G_{C}(s)$ a PI controller; and $\lambda=\mu=0$, a P controller. Without limiting $\lambda$ and $\mu$ to be 0 or 1 , fractional order offers more flexibility in the controller design.

The fractional order differentiator $s^{r}$ can be expressed as $s=w\left(z^{-1}\right)$ by generating functions. Choosing the Tustin generating function, the fractional differointegral can be discretized as [6]

$$
\left(w\left(z^{-1}\right)\right)^{ \pm r}=\left(\frac{2}{T} \frac{1-z^{-1}}{1+z^{-1}}\right)^{ \pm r}
$$

Expand $\left(\frac{1-z^{-1}}{1+z^{-1}}\right)^{r}$ in Taylor series as follows:

$$
\begin{aligned}
& \left(\frac{1-z^{-1}}{1+z^{-1}}\right)^{r} \\
& =1-(2 r) z^{-1}+\left(2 r^{2}\right) z^{-2}-\left(\frac{2}{3} r+\frac{4}{3} r^{3}\right) z^{-3} \\
& +\left(\frac{4}{3} r^{2}+\frac{2}{3} r^{4}\right) z^{-4}-\left(\frac{2}{5} r+\frac{4}{3} r^{3}+\frac{4}{15} r^{5}\right) z^{-5}+\cdots
\end{aligned}
$$

Therefore,

$$
\begin{aligned}
& s^{r}=\left(\frac{2}{T}\right)^{r}\left(1-(2 r) z^{-1}+\left(2 r^{2}\right) z^{-2}-\left(\frac{2}{3} r+\frac{4}{3} r^{3}\right) z^{-3}\right. \\
& \left.+\left(\frac{4}{3} r^{2}+\frac{2}{3} r^{4}\right) z^{-4}-\left(\frac{2}{5} r+\frac{4}{3} r^{3}+\frac{4}{15} r^{5}\right) z^{-5}+\cdots\right) \\
& \approx\left(\frac{2}{T}\right)^{r} \frac{A_{n}\left(z^{-1}, r\right)}{A_{n}\left(z^{-1},-r\right)}
\end{aligned}
$$

where the numerator $A_{n}\left(z^{-1}, r\right)$ and the denominator $A_{n}\left(z^{-1},-r\right)$ can be found by the following iterations.

$$
\begin{aligned}
& A_{0}\left(z^{-1}, r\right)=1 \\
& A_{n}\left(z^{-1}, r\right)=A_{n-1}\left(z^{-1}, r\right)-c_{n} z^{-n} A_{n-1}(z, r)
\end{aligned}
$$

where $c_{n}=\left\{\begin{array}{cc}r / n, & n \text { is odd } \\ 0, & n \text { is even }\end{array}\right.$
The accuracy of the approximation is determined by $n$.

\subsection{Controller Parameters}

Since the integer PID controller can be considered as a special case of the $\mathrm{PI}^{\lambda} \mathrm{D}^{\mu}$ controller, we can use the parameters determined by the Ziegler-Nichols method as a reference to obtain proper parameters of the $\mathrm{PI}^{\lambda} \mathrm{D}^{\mu}$ controller.

According to the Ziegler-Nichols method, $K_{u}$ and $T_{u}$ at fixed amplitude oscillation should be found first. The RouthHurwitz criterion [7] was used to find the value $K_{u}$. The characteristic equation of the motor system is

$$
L_{a} J s^{3}+\left(J R_{a}+B L_{a}\right) s^{2}+\left(R_{a} B+K_{t} K_{b}\right) s+K_{t} K_{u}=0
$$

Hence, the Routh-Hurwitz table was established.

$\begin{array}{ccc}s^{3} & L_{a} J & R_{a} B+K_{t} K_{b} \\ s^{2} & \left(J R_{a}+B L_{a}\right) & K_{t} K_{u} \\ s^{1} & b_{1} & 0 \\ s^{0} & K_{t} K_{u} & 0\end{array}$

where $b_{1}=\frac{\left(R_{a} B+K_{t} K_{b}\right)\left(J R_{a}+B L_{a}\right)-L_{a} J K_{t} K_{u}}{J R_{a}+B L_{a}}$

The system becomes unstable when $b_{1} \leq 0$. The critical condition $b_{1}=0$ yields $K_{u}=16.998$ at resonance. And the simulated step response is shown in Fig. 3.

The oscillation period of the controlled system in Fig. 3 is $T_{u}=0.15326$. The PID parameters determined by the ZieglerNichols method are $K_{P}=0.6 K_{u}=10.1988, K_{I}=2 K_{P} / T_{u}=$ 133.09434, and $K_{D}=K_{P} T_{u} / 8=0.19538$.
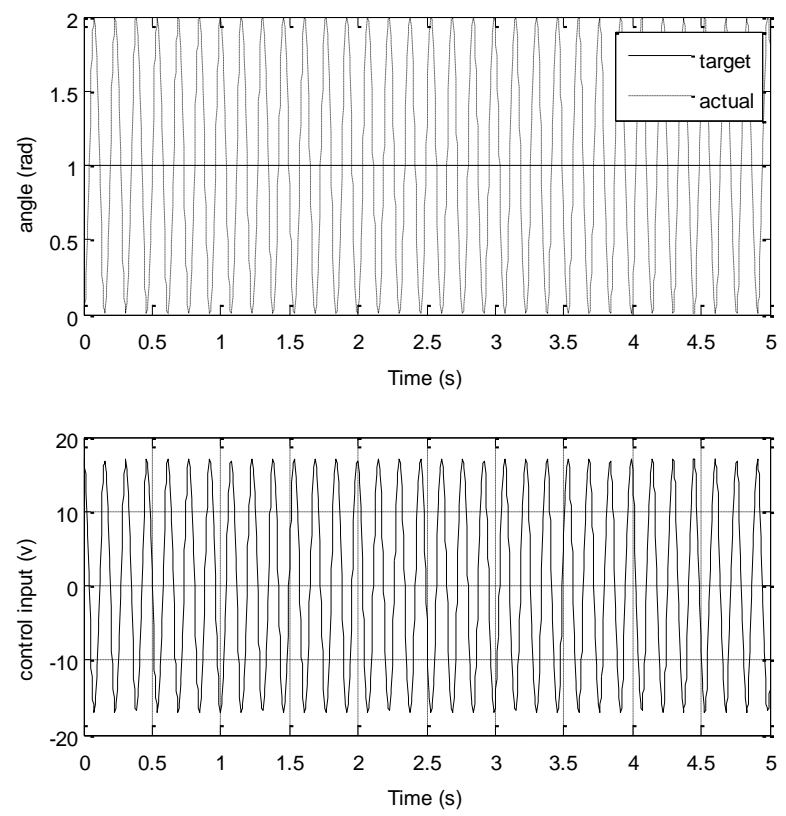

Figure 3. Step response and control input at $K=16.998$. 


\section{Iv. Simulation Results}

The proportional gain, integral gain and derivative gain found using the Ziegler-Nichols method are used in the PID controller and served as a basis for determining the parameters of the $\mathrm{PI}^{\lambda} \mathrm{D}^{\mu}$ controllers in this paper. A one-radian step input was used to compare the performance of the controllers. For the standard PID controller, $K_{P}=10.2, K_{I}=133$, and $K_{D}=$ 0.19 . The step response of integer PID controller and the corresponding control input are shown in Fig. 4. The maximum overshoot was about $0.825 \mathrm{rad}$., and the output settled to $\pm 5 \%$ window at $t=0.76 \mathrm{~s}$.

First, $\lambda=\mu=0.8$ (close to 1 ) was tried. The adopted parameters were $K_{P}=10.2, K_{I, \lambda}=80$, and $K_{D, \mu}=0.43$. The step response of the $\mathrm{PI}^{\lambda} \mathrm{D}^{\mu}$ controller with $\lambda=\mu=0.8$ and the corresponding control input are shown in Fig. 5. The maximum overshoot was about 0.79 rad., and the settling time was about $0.675 \mathrm{~s}$. The response didn't differ much from the integer PID controller's. Then $\lambda=\mu=0.5$ was used for the $\mathrm{PI}^{\lambda} \mathrm{D}^{\mu}$ controller. With $K_{P}=10.2, K_{I, \lambda}=36.84$, and $K_{D, \mu}=1.4$, the step response of the $\mathrm{PI}^{\lambda} \mathrm{D}^{\mu}$ controller with $\lambda=\mu=0.5$ and the corresponding control input are shown in Fig. 6. The maximum overshoot was about 0.93 rad., and the settling time was about $2.725 \mathrm{~s}$. The response showed that its performance was worse than the integer PID controller's. Since larger derivative gain might effectively reduce the oscillation amplitude for integer PID controller, the concept should apply to fractional order PID controller as well. By setting $K_{D, \mu}=2.8$ without changing $K_{P}$ and $K_{I, \lambda}$, the step response and the corresponding control input are shown in Fig. 7. The maximum overshoot was about $0.8 \mathrm{rad}$., and the settling time was about $0.685 \mathrm{~s}$. And the performance was better than the integer PID controller's, which verified the assumption about the fractional derivative gain.

Furthermore, $K_{D, \mu}=7$ was chosen without changing $K_{P}$ and $K_{I, \lambda}$ for $\mathrm{PI}^{\lambda} \mathrm{D}^{\mu}$ controller with $\lambda=\mu=0.5$. The step response and the corresponding control input are shown in Fig. 8. The maximum overshoot was about 0.66 rad., and the settling time was about $0.272 \mathrm{~s}$. The performance of this controller was far better than the integer PID controller's performance with the empirical gains. Because, the empirical gains obtained by the Ziegler-Nichols method might not be optimal for the integer PID controller, more vigorous researches may be conducted to judge the condition which controller prevails. At this point, at least the $\mathrm{PI}^{\lambda} \mathrm{D}^{\mu}$ controller may work better if the gains were appropriately chosen.
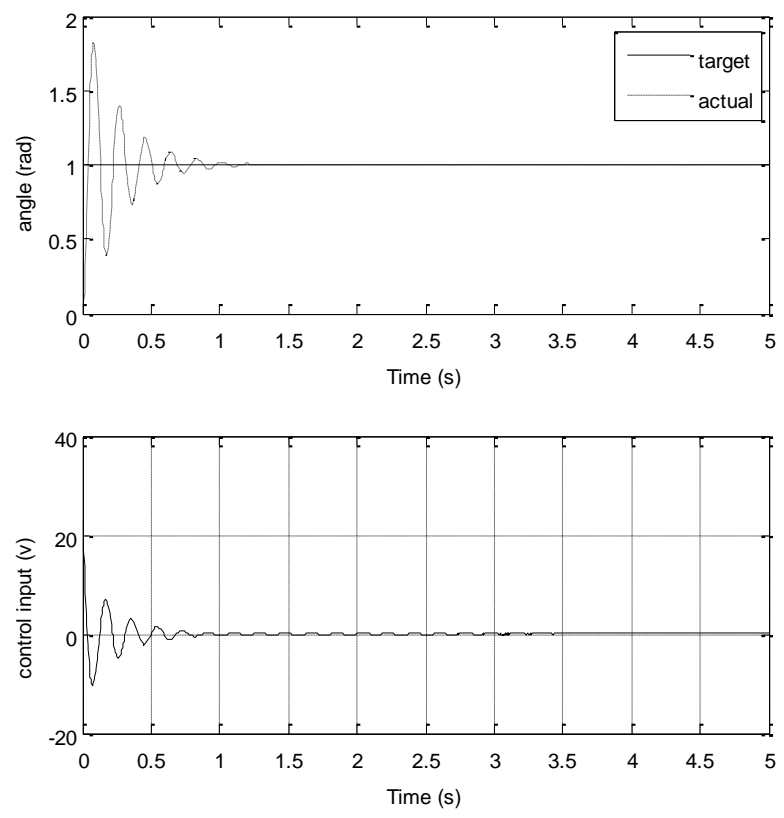

Figure 4. Step response and control input under PID control.
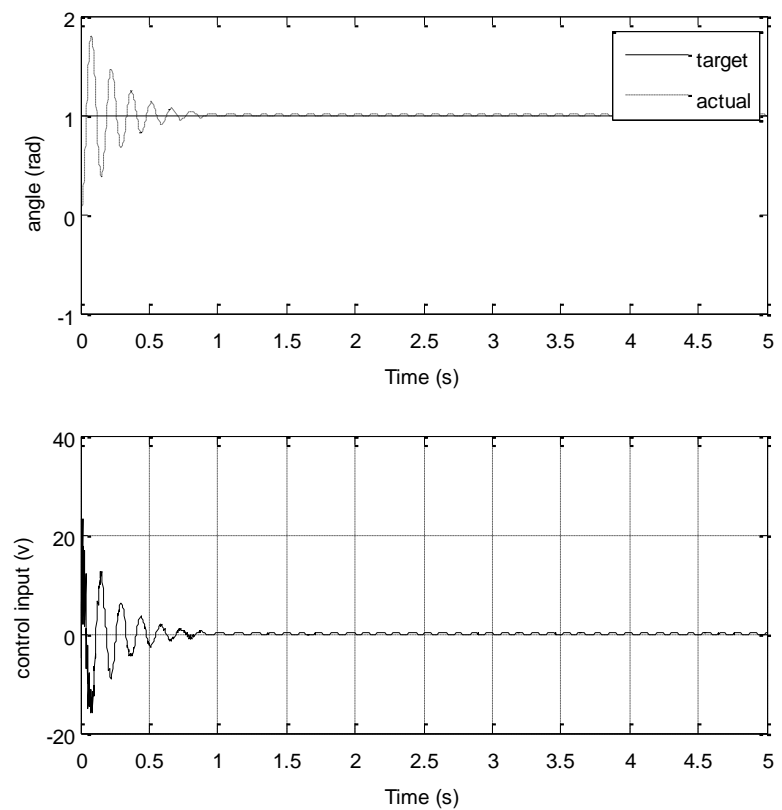

Figure 5. Step response and control input under $\mathrm{PI}^{\lambda} \mathrm{D}^{\mu}$ control $(\lambda=\mu=0.8)$ 

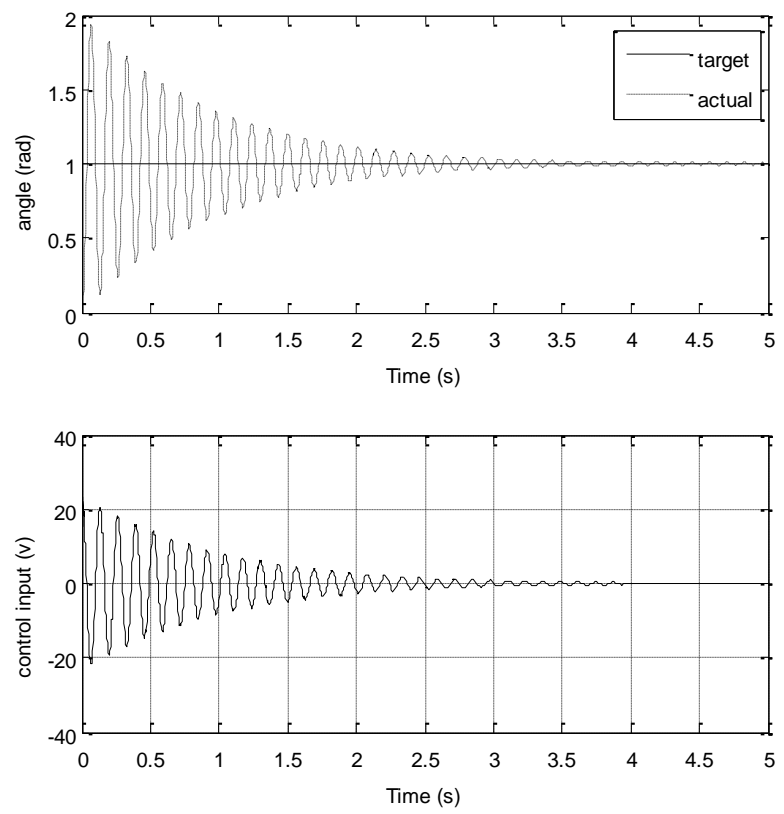

Figure 6. Step response and control input under $\operatorname{PI}^{\lambda} \mathrm{D}^{\mu} \operatorname{control}(\lambda=\mu=0.5)$ $\left(\lambda=\mu=0.5, K_{D, \mu}=1.4\right)$
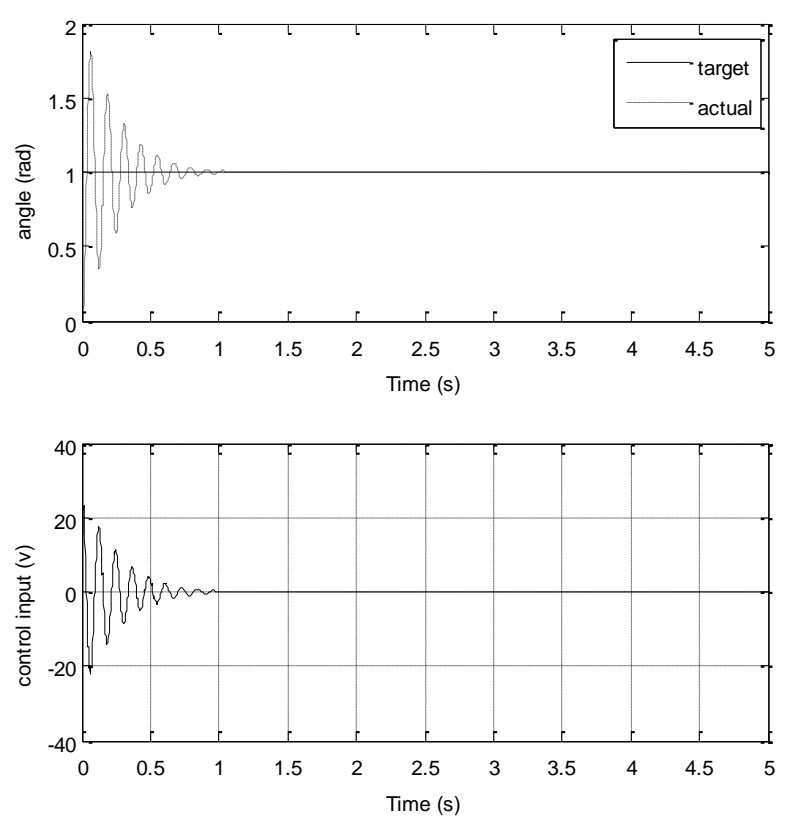

Figure 7. Step response and control input under $\mathrm{PI}^{\lambda} \mathrm{D}^{\mu}$ control $\left(\lambda=\mu=0.5, K_{D, \mu}=2.8\right)$
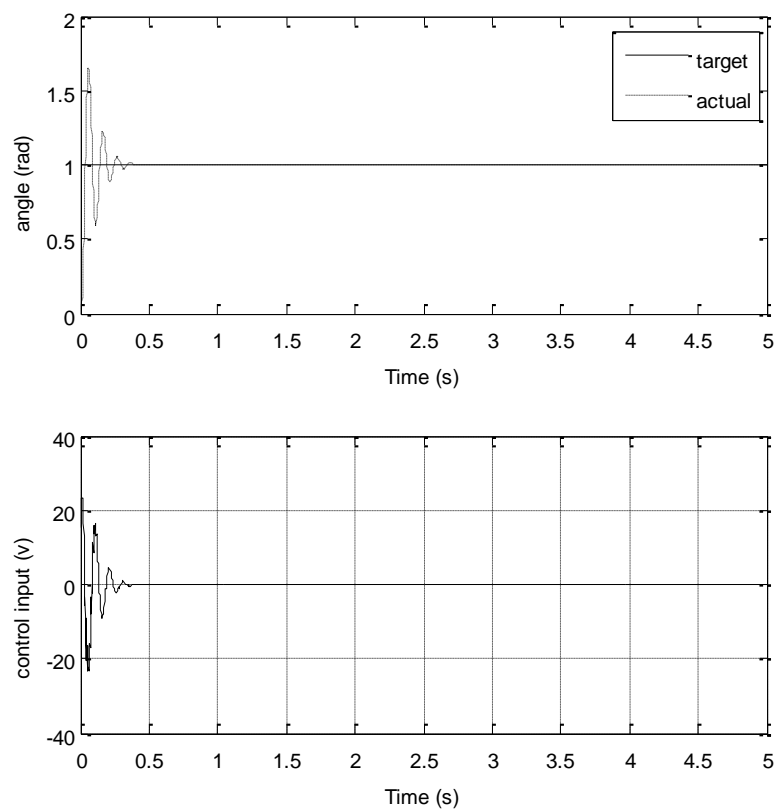

Figure 8. Step response and control input under $\mathrm{PI}^{\lambda} \mathrm{D}^{\mu}$ control $\left(\lambda=\mu=0.5, K_{D, \mu}=7\right)$

\section{v. Conclusions}

Different fraction numbers were used in $\mathrm{PI}^{\lambda} \mathrm{D}^{\mu}$ controllers for comparison with (integer) PID controller in the case of a DC motor system. The simulation results showed that as $\lambda$ and $\mu$ approached integer 1 , the fractional order PID controller worked like a PID controller as expected. In addition, the case $\lambda=\mu=0.5$ was studied for comparison. These results show the fractional order controller is promising and may work better than the (integer) PID controller.

\section{References}

[1] K. S. Miller and B. Ross, "An Introduction to the Fractional Calculus and Fractional Differential Equations," New York: Wiley, 1993.

[2] Concepcion A. Monje, Blas M. Vinagre, Vicente Feliu, YangQuan Chen, "Tuning and auto-tuning of fractional order controllers for industry applications," Control Engineering Practice, 16, pp. 789-812, Oct. 2007.

[3] Durate Valério, Jose Sá da Costa, "Tuning of fractional PID controllers with Ziegler-Nichols-type rules," Science Signal Processing, 86, pp. 2771-2784, Mar. 2006.

[4] Hongmei Fan, Yu Sun and Xiaobin Zhang, "Research on Fractional Order Controller in Servo Press Control System," IEEE International Conference on Mechatronics 2007.

[5] A. Oustaloup, "La Derivation non Entiere," Paris, France: Hermes, 1995.

[6] Y. Q. Chen and K. L. Moore, "Discretization schemes for fractional order differentiators and integrators," IEEE Trans. Circuits Syst., Vol. 49, No. 3, pp. 363-367, 2002

[7] Norman S. Nise, "Control Systems Engineering 6th edition," New York: Wiley, 2010. 